\title{
Evaluación de la Gestión del Conocimiento en las Organizaciones de la Red de Tecnologías de Información y Comunicaciones del Eje Cafetero en Colombia
}

\author{
Carlos E Marulanda ${ }^{(1)}$, Jaime A Giraldo ${ }^{(1)}$ y Marcelo López ${ }^{(2)}$ \\ (1) Universidad Nacional de Colombia, Facultad de Ingeniería, Departamento de Ingeniería Industrial. \\ Campus Palogrande. Calle 27 No. 64-60, Manizales-Colombia. (e-mail: cemarulandae@unal.edu.co, \\ jaiagiraldog@unal.du.co). \\ (2) Universidad de Caldas, Facultad de Ingeniería, Departamento de Sistemas e Informática. \\ Calle 65 No. 26 -10, Manizales-Colombia. (e-mail: mlopez@ucaldas.edu.co)
}

Recibido Nov. 28, 2012; Aceptado Ene. 31, 2013; Versión final recibida Mar. 14, 2013

\begin{abstract}
Resumen
El objetivo del trabajo fue establecer el estado de la gestión del conocimiento en la red de tecnologías de información y comunicaciones de la región Eje Cafetero de Colombia. Se evaluaron las prácticas y competencias de los empleados para gestionar el conocimiento en veintidós empresas cafeteras. Se evidencian avances en el desarrollo de las competencias para la gestión del conocimiento, así como un lento avance en la apropiación y uso de prácticas. Se propone a las empresas incorporar el manejo direccionado de procesos de conocimiento. Este estudio aporta a la comunidad académica y a la red cafetera una forma de evaluación y genera una línea base para potenciar el capital intelectual y la gestión del conocimiento organizacional.
\end{abstract}

Palabras clave: gestión de conocimiento, buenas prácticas, TIC, competencias personales

\section{Evaluation of Knowledge Management in Organizations of the Information and Communication Technology Network of the Coffee Industry in Colombia}

\begin{abstract}
The aim of this work was to establish the state of knowledge management in the cluster of information and communications technologies in the coffee region of Colombia. Twenty two companies were evaluated in relation to practice, and skills of their employees to manage knowledge. Progress is evident in the development of skills for knowledge management and slow progress on the appropriation and use of practices. It is proposed that companies incorporate a guided knowledge management policy. This study contributes to the academic community and to the coffee cluster with a new form of evaluation and a baseline to enhance intellectual capital and organizational knowledge management.
\end{abstract}




\section{INTRODUCCIÓN}

Según Toffler (1990), la evolución empresarial se ha dado en una primera ola, por una sociedad agraria marcada por la ocupación y el uso de la energía muscular, la segunda civilización u ola, por una sociedad industrial, caracterizada por el modo de producción masiva, el dinero y la energía electromecánica y la tercera civilización, por la sociedad de la información, identificada por el conocimiento y la electrónica.

Para (Alonso, 2006), (Capó-Vicedo et al., 2007), la sociedad actual se caracteriza por la transición de la gestión de personas, la flexibilidad de las acciones empresariales, la incertidumbre por los mercados desregulados y la formación de una economía interconectada, lo que incide en que las empresas, estén sujetas a cambios que ocasionan la aparición de nuevos empresarios, productos y servicios con un menor ciclo de vida, el uso de las tecnologías de información y comunicaciones (TIC), el desarrollo de la telefonía y computación móvil, los servicios orientados a clientes, la mercadotecnia y la innovación.

En este contexto, las empresas tienen la exigencia de generar y fortalecer las ventajas competitivas sostenibles y sustentables para responder, adaptar y sostenerse (Porter, 1991), (Alvarez, 2004), (OCDE, 1997), (Matilla y Chalmeta, 2007) y lo han hecho basados en diversos enfoques, que van desde la gestión del conocimiento, la incorporación a un clúster (Sanabria et al., 2010), la innovación y las TIC (Romero et al., 2009) y el mejoramiento continuo (Meisel et al., 2006) entre otros.

El enfoque de la innovación debe ser parte vital de todos los sectores económicos y hacerse extensivo a todos sus eslabonamientos (DNP, 2005), (EUROSTAT, 2006), (Morales, 2010) y en el marco de la gestión del conocimiento es un agente que dinamiza externalidades en los sistemas productivos, (Hautala, 2011), (Knudsen, 1995), (Marulanda y Lopez, 2011), (López y Calderón, 2006), además de ser un factor fundamental de la competitividad.

Gestión de conocimiento que definen (Matos et al, 2006), como el proceso sistemático de buscar, organizar, filtrar y presentar la información con el objetivo de mejorar la comprensión de las personas en una específica área de interés. Y que se evalúa según (Marr, 2004), desde el capital intelectual (Cl); para el efecto (Sánchez, 2003), comenta que existen varios modelos para la medición del capital intelectual, de los más conocidos son: el modelo de Sullivan, el Capital Intelectual de Skandia, el modelo Brooking, el modelo de categorización de Roos, el modelo de Onge, el modelo de Sveiby y el modelo de Wiig, entre otros. Además de lo que establecen (Aguilera et al, 2011), que coinciden en señalar tres elementos que forman parte del concepto de capital intelectual: el capital humano, el capital estructural y el capital relacional.

En cuanto a los esquemas desde la gestión del conocimiento, (Corbin et al., 2007), se enfocan en el desarrollo de prototipos, experimentación y evaluación del producto; (Andrade et al., 2008), la conceptualizan y representan de una manera sistemática, orientando la definición de una memoria corporativa; (Prassl et al., 2005), establecen un esquema para permitir a los usuarios actualizar los conocimientos, manejando la incertidumbre de fenómenos multidimensionales; (Bingi y Yadav, 1995), definen que se debe poseer una combinación adecuada de: métodos y requisitos de inferencia y transparencia en el control la integridad y la consistencia y (McBriara et al, 2003), relacionan la gestión del conocimiento con la capacidad de tomar decisiones informadas.

Una perspectiva que permitiría abordar la complejidad del análisis organizacional y de sus interacciones a través de una forma de pensamiento basada en la totalidad y sus propiedades (Daedalus, 2009), (Bueno, 2003), (Chiva-G, 2004), tal como se observa en los modelos planteados por (Peña, 2006), (Riesco, 2006) y (Pérez, 2007). Pero desde estos modelos y esquemas, no se considera el desarrollo organizacional basada en conocimiento, conjuntando enfoques como son: la gestión estratégica, la gestión por competencias y la gestión por procesos. Para este caso en particular las organizaciones determinaran su estado y evolución como gestores de conocimiento mediante una evaluación integral y permanente de las siguientes categorías: análisis organizacional, capacitación, competencias, estrategia, indicadores, modelo de madurez, prácticas, procesos, sistema y tecnologías de información y comunicaciones TI.

Se han hecho algunos estudios relacionados, como es el caso de las siguientes tesis doctorales realizadas en España: la caracterización del conocimiento en PYMES que realizan proyectos TIC: un modelo de análisis y valoración (Lobato, 2008) y el desarrollo de un modelo de gestión del conocimiento en la cadena de suministro de la industria agroalimentaria (Martínez, 2011).

En Colombia se observan tendencias de acuerdo a los resultados de investigaciones relacionadas con: el grado de coincidencia entre el concepto y el enfoque que tienen las organizaciones sobre la gestión del conocimiento (Briceño y Bernal, 2010), la valoración de la sociedad del conocimiento (Marulanda y López, 2011), la identificación del aprendizaje organizacional y la generación de capital intelectual en la gestión del 
conocimiento (Garzón C., 2006), (Paniagua, 2007) y (Carrillo, F., 2005), la generación de valor y el fortalecimiento de las ventajas competitivas de las compañías (Bernal et al., 2010).

En ese sentido el sector de TIC del eje cafetero colombiano, en el año 2011, según Patria (2011), concluyó la iniciativa de 22 empresas y organizaciones para conformar el clúster, buscando acceder a oportunidades y mercados de mayor envergadura. Dicho sector según Valencia (2010), se caracteriza por la alta concentración en el mercado interno, por un bajo nivel de asociatividad y la escasez de recursos financieros.

Pero según Proexport (2011), el sector de TIC en el país es uno de los más promisorios y se caracteriza porque: es tercero en América Latina; es un mercado con un amplio potencial de penetración; los ingresos se han casi duplicado desde 2005; la industria emplea aproximadamente a 168.000 personas; se cuenta con la tasa de piratería más baja de la región y su calidad y los costos de operación son altamente competitivos.

En una caracterización del sector de TI del triángulo del café, (Joyanes et al., 2010) se evidenció que el "tipo de producto o servicio" que más ofrecen las empresas del eje cafetero a sus clientes tiene que ver con: "software a la medida", "software empaquetado" y "aplicaciones web". Los sectores que más demanda presentan son: los de servicios, comercio, industria y de gobierno con porcentajes respectivos de $16.23 \%$, $14.04 \%, 11.84 \%$ y $11.40 \%$.

Lo anteriormente expuesto reconoce la capacidad de desarrollo del sector para satisfacer las necesidades de los clientes locales, regionales y nacionales, pero con la gestión del conocimiento serían mejores los resultados, tal como lo establecen (Torres, 2006), (Kok, 2007), (Shin et al., 2001), (Johannessena et al., 2005), (Nahapiet y Ghoshal, 1998).

Es por esto que se hace necesario conocer el estado de la gestión del conocimiento en las empresas del clúster; para el efecto se evaluaron dichas empresas en las categorías de: competencias y prácticas para la gestión del conocimiento, en el marco de la investigación doctoral "Desarrollo de un modelo de evaluación de la gestión del conocimiento en PYMES del sector TI del eje cafetero", que se fundamenta en un método de investigación exploratoria, descriptiva y correlacional.

\section{MATERIALES Y MÉTODOS}

Para el desarrollo se utilizó desde una investigación exploratoria, para analizar e identificar las principales variables del modelo de evaluación, pasando por una investigación descriptiva, para conocer las situaciones predominantes con la representación exacta de los procesos, hasta una investigación de tipo correlacional para encontrar la correlación entre las variables.

\section{Muestreo}

Se aplicó la encuesta a través de internet a la totalidad de la población, 22 organizaciones y empresas del clúster de TIC de la región del Eje Cafetero de Colombia. Previa la valoración, se desarrollo una prueba piloto de la encuesta con expertos sobre el tema, además de una revisión con algunos de los actores del clúster.

Para la estructuración del cuestionario se formularon preguntas en escala Likert, las cuales se calificaron de 1 a 5, donde 1, se está en desacuerdo o no realizado, 2, realizado parcialmente, 3, realizado en intervalos de tiempo, 4 , realizado con regularidad y 5 realizado completamente.

\section{Categorías evaluadas}

Las categorías y variables se fundamentan en los modelos y planteamientos desde la perspectiva europea, (CEN, 2004), el foro europeo de conocimiento, (Chen, 2008), y los desarrollos de autores como (Bueno, 2003), (DelMoral et al., 2007) y (López M. , 2010), en los que se define la medición desde el capital humano, organizativo, tecnológico, relacional e intangible, entre otros, considerando las interrelaciones y las perspectivas dinámicas de una organización del conocimiento, por medio de métricas, herramientas e indicadores específicos, ligados a las competencias individuales y las capacidades organizacionales. Estas se agruparon en 77 preguntas (ver tabla 1): 
Tabla 1: Categorías y variables a evaluar. Fuente: elaboración propia

\begin{tabular}{|l|l|c|}
\hline \multicolumn{1}{|c|}{ Categorías } & \multicolumn{1}{|c|}{ Variables } & $\begin{array}{c}\text { No. } \\
\text { Preguntas }\end{array}$ \\
\hline Competencias & $\begin{array}{l}\text { Gestión de la información y la documentación, gestión de la comunicación, diseño } \\
\text { de herramientas digitales, gestión de la innovación y el cambio y gestión del } \\
\text { aprendizaje organizacional. }\end{array}$ & $\begin{array}{c}44 \\
\text { Prácticas }\end{array}$ \\
\hline
\end{tabular}

Para revisión de la herramienta de encuesta y los resultados, por favor ingresar al portal www.logopoliskm.com/logomanager, con el usuario: carloselog@gmail.com y la contraseña: carlose1999.

Las variables medidas, en la categoría competencias se componen de:

Gestión de la información y la documentación, en lo referente a: análisis de requerimientos de información, gestión documental, gestión digital de archivos, bibliotecas y servicios, vigilancia tecnológica (para alertar sobre cualquier innovación científica o técnica susceptible de crear oportunidades o amenazas (Escorsa, 2001), (Klavans, 1993), (Zaintek, 2003), (Tena, 2006) y (Palop, 1999), como factor fundamental para el avance de las organizaciones de la actualidad).

Gestión de la comunicación, en lo referente a: diseño de entornos web, materiales digitales, boletines de noticias, alertas y tablones virtuales, edición digital de revistas, sondeos y encuestas de clima organizacional, diseño físico y digital de espacios corporativos.

Gestión de la innovación y el cambio, en lo referente a: análisis y rediseño de procesos, diseño de servicios y productos, sistemas de evaluación del desempeño, sistema de gestión por objetivos, proyectos y programas de I+D+i (Investigación, Desarrollo e innovación), protección de datos y capital intelectual, gestión del cambio y del riesgo

Diseño de herramientas digitales, en lo referente a: sistemas de taxonomías digitales, mapas digitales de conocimiento, análisis de usabilidad de las TIC, desarrollo de contenidos digitales, formación y capacitación en el uso y apropiación de las TIC.

Gestión del aprendizaje organizacional, en lo referente a: análisis de las redes sociales, gestión de proyectos, diseño de sistemas de detección, análisis y comunicación de las mejores prácticas, comunidades de práctica y grupos de discusión, planes de formación y capacitación, sistema de gestión por competencias, trabajo colaborativo y gestión de calidad.

Las variables medidas, en la categoría prácticas se componen de:

Identificación de conocimiento, en lo referente a: lecciones aprendidas, mapas de conocimiento, cuadro de mando integral, evaluación de patentes y portafolio de conocimientos.

Generación de conocimiento en lo referente a: sistematizar y comunicar las mejoras practicas, uso de tormenta de ideas, círculos de conocimiento, grupo de expertos, benchmarking interno, benchmarking externo, esquema digital de sugerencias y sistema de gestión de la formación.

Compartición de conocimiento en lo referente a: sistemas de videoconferencia, foros dirigidos de discusión, diario digital del empleado, pizarrón corporativo intranet, acceso a una literatura especializada y mentoring corporativo

Retención de conocimiento en lo referente a: sistemas de gestión documental, sistema de páginas amarillas, sistemas de expertos, centros digitales de información y cuadernos explicativos.

Aplicación de conocimiento en lo referente a: ferias internas de conocimiento, bróker de conocimiento, sistema de tecnología scout, sistemas digitales de ayuda, programas de gestión del tiempo, coaching corporativo y entornos de trabajo colaborativo

\section{RESULTADOS Y DISCUSIÓN}

El proceso de recolección de datos se hizo de manera personal y telefónica con los encargados de las empresas en el momento del registro, considerando que tuvieran comprensión de procesos relacionados con la gestión del conocimiento organizacional, además de hacer una posterior revisión de las respuestas y 
un muestreo aleatorio de comprobación. Los resultados consolidados de las categorías evaluadas se pueden observar en la tabla 2 .

Tabla 2: Resultados de la encuesta. Fuente: elaboración propia

\begin{tabular}{|c|c|c|c|c|c|c|c|c|c|c|}
\hline \multirow[b]{2}{*}{ Empresas / variables de medición } & \multicolumn{5}{|c|}{ Competencias } & \multicolumn{5}{|c|}{ Prácticas } \\
\hline & 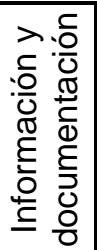 & 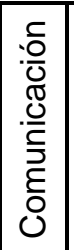 & 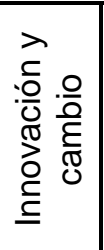 & 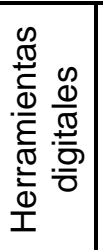 & 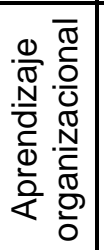 & 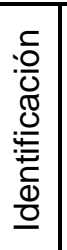 & 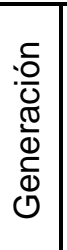 & 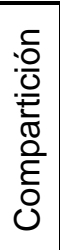 & 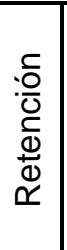 & 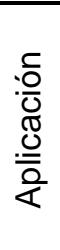 \\
\hline Alsus IT group & 5 & 5 & 5 & 4 & 5 & 3 & 4 & 4 & 5 & 3 \\
\hline Apolo & 5 & 5 & 5 & 5 & 5 & 4 & 4 & 5 & 4 & 4 \\
\hline Cámara de comercio de Armenia & 5 & 5 & 5 & 5 & 5 & 5 & 5 & 5 & 5 & 5 \\
\hline Cámara de comercio de Manizales & 4 & 4 & 3 & 3 & 4 & 4 & 4 & 3 & 4 & 3 \\
\hline Cámara de comercio de Pereira & 5 & 5 & 5 & 5 & 3 & 2 & 3 & 2 & 2 & 3 \\
\hline Controlsoft & 0 & 0 & 0 & 0 & 0 & 0 & 0 & 0 & 0 & 0 \\
\hline EAM & 4 & 4 & 4 & 4 & 4 & 4 & 4 & 4 & 4 & 4 \\
\hline Heinsohn & 5 & 5 & 5 & 5 & 5 & 5 & 5 & 5 & 4 & 5 \\
\hline Insoft Ltda. & 5 & 5 & 5 & 5 & 5 & 2 & 3 & 5 & 2 & 5 \\
\hline Mangalink & 5 & 5 & 5 & 5 & 5 & 5 & 5 & 4 & 5 & 1 \\
\hline ParqueSoft Manizales & 5 & 5 & 4 & 4 & 5 & 3 & 3 & 3 & 2 & 3 \\
\hline ParqueSoft Armenia & 5 & 5 & 5 & 5 & 5 & 4 & 5 & 5 & 5 & 4 \\
\hline Parquesoft Pereira & 5 & 5 & 5 & 5 & 5 & 3 & 4 & 3 & 3 & 4 \\
\hline PGC Ltda. & 5 & 5 & 5 & 5 & 4 & 4 & 3 & 1 & 2 & 4 \\
\hline Quántica & 3 & 3 & 3 & 3 & 3 & 3 & 3 & 3 & 3 & 3 \\
\hline SIA & 3 & 3 & 4 & 3 & 3 & 3 & 4 & 3 & 3 & 3 \\
\hline Sigma ingeniería & 5 & 5 & 5 & 5 & 5 & 4 & 4 & 2 & 2 & 2 \\
\hline SUMSET & 5 & 5 & 5 & 5 & 5 & 3 & 5 & 1 & 1 & 1 \\
\hline Tecnología y software & 4 & 4 & 3 & 3 & 3 & 3 & 3 & 1 & 2 & 3 \\
\hline Universidad Autónoma & 5 & 5 & 5 & 5 & 5 & 3 & 3 & 3 & 2 & 3 \\
\hline Universidad Católica & 5 & 5 & 4 & 5 & 4 & 4 & 4 & 3 & 4 & 5 \\
\hline Universidad Caldas & 2 & 3 & 2 & 3 & 2 & 2 & 3 & 3 & 2 & 2 \\
\hline
\end{tabular}

De estos resultados se resalta, luego de explicar con detalle cada una de las preguntas a los encuestados, puntajes diferenciales, como es el caso de la empresa Controlsoft, la cual tiene un puntaje de 0 en todas sus respuestas, o el caso de los puntajes de 1 de la empresa Sumset y 2 en la Universidad de Caldas, además el contraste extremo que se dan por las calificaciones de 5 en varias de las respuestas. De todas formas el trabajo estadístico permite que los resultados extremos no se consideren en el ejercicio de los cálculos correspondientes.

Se presenta igualmente la matriz de correlación, ver tabla 3, la cual muestra un alto grado de correlación entre las variables evaluadas, de tal manera que es posible aplicar el método de componentes principales y así en algunos factores consolidar la información de competencias y prácticas en gestión del conocimiento de las organizaciones del clúster de TIC del Eje Cafetero de Colombia. 
Tabla 3: Tabla de correlaciones (valor p). Fuente: elaboración propia

\begin{tabular}{|c|c|c|c|c|c|c|c|c|c|c|}
\hline & 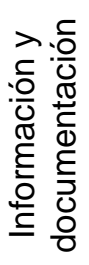 & 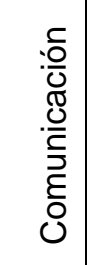 & 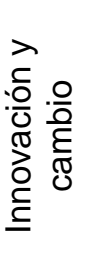 & 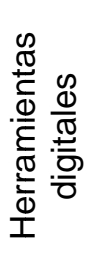 & 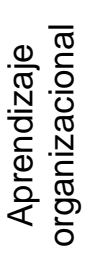 & 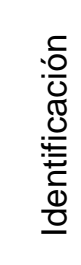 & $\begin{array}{l}\frac{\sigma}{0} \\
\frac{\pi}{U} \\
\frac{\pi}{0} \\
\frac{5}{0} \\
0\end{array}$ & 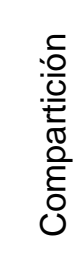 & 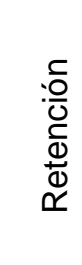 & $\begin{array}{l}\frac{0}{0} \\
\frac{0}{0} \\
\frac{0}{0} \\
\frac{0}{4}\end{array}$ \\
\hline Información y documentación & & ,000 & ,000 & ,000 & ,000 & ,308 & ,048 & ,490 & 899 & ,430 \\
\hline Comunicación & 000 & &, 000 & 000 & 000 & ,324 & ,046 &, 514 & ,931 & ,435 \\
\hline Innovación y cambio & ,000 & 000 & & 000 & 000 & ,283 & 021 & ,372 & 810 &, 570 \\
\hline Herramientas digitales &, 000 & 000 &, 000 & &, 000 & ,224 & ,031 &, 582 & ,908 & ,366 \\
\hline Aprendizaje organizacional & 000 & 000 & 000 & ,000 & & ,094 & ,004 & 070 & ,431 & ,642 \\
\hline Identificación & ,308 & 324 & ,283 & ,224 & ,094 & & ,000 & ,096 & ,001 & ,448 \\
\hline Generación & 048 & ,046 & ,021 & 031 & ,004 & ,000 & & ,076 & ,006 & ,984 \\
\hline Compartición & 490 &, 514 & ,372 & ,582 & ,070 & ,096 & ,076 & & ,000 & ,017 \\
\hline Retención & 899 & ,931 & 810 & 908 & 431 & 001 & ,006 & ,000 & & , 168 \\
\hline Aplicación & 430 & 435 & ,570 & ,366 & 642 & 448 & ,984 & ,017 & 168 & \\
\hline
\end{tabular}

Desde la categoría competencias para la gestión del conocimiento, los resultados se graficaron tal como se observa en la figura 1.

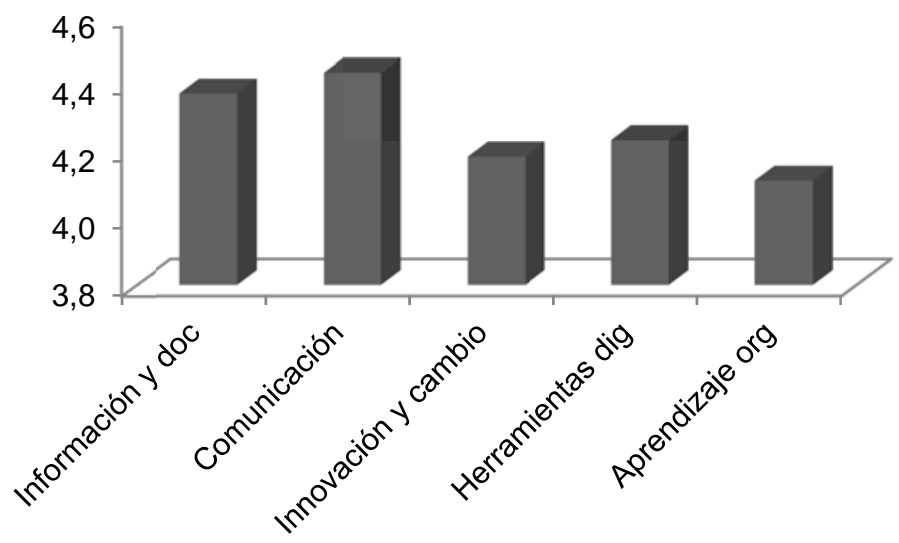

Fig. 1: Competencias para la gestión del conocimiento Fuente: elaboración propia a partir de encuesta 2.0.

De acuerdo a la escala de calificación, el promedio de las variables evaluadas está alrededor de 4.3, lo que muestra un avance significativo en el desarrollo de las competencias para la gestión del conocimiento, las cuales posibilitan la conciliación y el apalancamiento del conocimiento individual (Barnes y Liao, 2012), además del aprovechamiento de herramientas, para la innovación de procesos, productos y servicios, necesario para una mayor competitividad.

Se contrasta en este caso como el aprendizaje organizacional, conjuntamente con la innovación, que se evidencia a manera de la primera magnitud de la fuerza competitiva (Camisóna y Monfort-Mir, 2012), y el cambio, tienen una valoración por debajo del promedio, considerando la importancia de estas variables en el marco de las competencias para la gestión del conocimiento, mientras que la comunicación es la más valorada, lo que hace necesario investigar con mayor profundidad estas variables, a fin de establecer estrategias para su mejoramiento.

Desde la categoría prácticas para la gestión del conocimiento, los resultados se graficaron tal como se observa en la figura 2. 


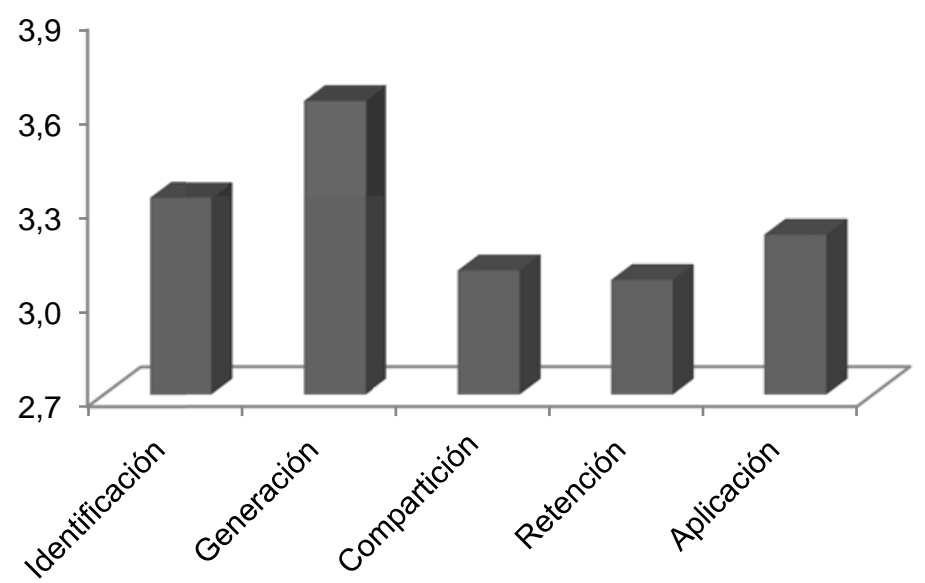

Fig. 2: Prácticas para la gestión del conocimiento Fuente: elaboración propia a partir de encuesta 2.0.

De acuerdo a la escala de calificación, el promedio de las variables evaluadas: identificación del conocimiento, generación de conocimiento, retención de conocimiento, compartición de conocimiento y aplicación de conocimiento, está alrededor de 3.3, lo que muestra, y en contraste con las competencias, que falta avanzar en la apropiación y uso de prácticas para la gestión del conocimiento, sobretodo en aspectos de orden cultural como lo es, el compartir y retener las prácticas con la empresa, para el beneficio de los mismos empleados.

Se contrasta en este caso la retención, conjuntamente con la compartición de conocimiento, tienen una valoración por debajo del promedio, considerando la importancia de estas variables en el marco de las prácticas para la gestión del conocimiento, mientras que la generación es la más valorada, lo que hace necesario investigar con mayor profundidad estas variables, a fin de establecer estrategias para su mejoramiento.

Dada la cantidad de variables que se evaluaron, se realizó un análisis de componentes principales, con el software estadístico SPSS para determinar, cuáles de ellas explican la gestión del conocimiento en la región. Lo que hace el software es analizar las variables y agruparlas en componentes sin asignar nombres, ver tabla 4.

Tabla 4: Componentes principales. Fuente: elaboración propia

\begin{tabular}{|c|r|r|r|r|r|r|}
\hline \multirow{2}{*}{ Componente } & \multicolumn{4}{|c|}{ Autovalores iniciales } & \multicolumn{3}{|c|}{ Sumas de las saturaciones al cuadrado-extracción } \\
\cline { 2 - 7 } & \multicolumn{1}{|c|}{ Total } & \% varianza & \% acumulado & Total & \% varianza & \% acumulado \\
\hline 1 & 5,173 & 51,727 & 51,727 & 5,173 & 51,727 & 51,727 \\
2 & 2,366 & 23,664 & 75,391 & 2,366 & 23,664 & 75,391 \\
3 & 1,173 & 11,726 & 87,116 & 1,173 & 11,726 & 87,116 \\
4 &, 547 & 5,466 & 92,582 & & & \\
5 &, 261 & 2,610 & 95,192 & & \\
6 &, 215 & 2,149 & 97,341 & & \\
7 &, 151 & 1,506 & 98,847 & & \\
8 &, 077 &, 774 & 99,620 & & \\
9 &, 031 &, 306 & 99,926 & & & \\
10 &, 007 &, 074 & 100,000 & & & \\
\hline
\end{tabular}

Con base en estos resultados (leer porcentaje acumulado de las líneas 1, 2 y 3) se establece lo siguiente: 
El primer componente explica el 51,7\% de los resultados, el segundo explica el $75,3 \%$ y el tercero explica el $87,1 \%$. Se procede entonces a asignar a cada variable evaluada un componente, de acuerdo al número mayor y como se observa en la tabla 5.

Tabla 5: Matriz de componentes. Fuente: elaboración propia

\begin{tabular}{|c|r|r|r|}
\hline \multirow{2}{*}{ Variables } & \multicolumn{3}{|c|}{ Componente } \\
\cline { 2 - 4 } & \multicolumn{1}{|c|}{1} & \multicolumn{1}{c|}{2} & \multicolumn{1}{c|}{3} \\
\hline Identificación & 0,499 & 0,613 & $-0,376$ \\
\hline Generación & 0,684 & 0,421 & $-0,474$ \\
\hline Compartición & 0,413 & 0,698 & 0,368 \\
\hline Retención & 0,313 & 0,874 & $-0,049$ \\
\hline Aplicación & 0,265 & 0,36 & 0,806 \\
\hline Información y documentación & 0,92 & $-0,336$ & 0,077 \\
\hline Comunicación & 0,919 & $-0,344$ & 0,075 \\
\hline Innovación y cambio & 0,912 & $-0,292$ & 0,02 \\
\hline Herramientas digitales & 0,896 & $-0,332$ & 0,059 \\
\hline Aprendizaje organizacional & 0,89 & $-0,07$ & $-0,055$ \\
\hline
\end{tabular}

El resultado de dicha asignación se observa en la tabla 6:

Tabla 6: Asignación. Fuente: elaboración propia

\begin{tabular}{|c|c|}
\hline Variable & $\begin{array}{c}\text { Representada por el factor de } \\
\text { componente }\end{array}$ \\
\hline Identificación & 2 \\
\hline Generación & 1 \\
\hline Compartición & 2 \\
\hline Retención & 2 \\
\hline Aplicación & 3 \\
\hline Información y documentación & 1 \\
\hline Comunicación & 1 \\
\hline Innovación y cambio & 1 \\
\hline Herramientas digitales & 1 \\
\hline Aprendizaje organizacional & 1 \\
\hline
\end{tabular}

Posteriormente se da nombre a estos factores, así:

FACTOR 1: GENERACIÓN DE CONOCIMIENTO

FACTOR: 2: IDENTIFICACIÓN DE CONOCIMIENTO

FACTOR 3: APLICACIÓN DE CONOCIMIENTO

Con estos factores se hace la matriz de correlaciones para corroborar que efectivamente los componentes están bien agrupados (ver tabla 7). Los resultados son los siguientes. 
Tabla 7: Correlaciones entre factores obtenidos. Fuente: elaboración propia

\begin{tabular}{|c|c|c|c|c|}
\hline & & $\begin{array}{l}\text { Puntuación de } \\
\text { factor } 1 \text { para } \\
\text { análisis } 1 \\
\end{array}$ & $\begin{array}{l}\text { Puntuación de } \\
\text { factor } 2 \text { para } \\
\text { análisis } 1 \\
\end{array}$ & $\begin{array}{l}\text { Puntuación de } \\
\text { factor } 3 \text { para } \\
\text { análisis } 1 \\
\end{array}$ \\
\hline \multirow{3}{*}{$\begin{array}{l}\text { Puntuación de factor } 1 \text { para } \\
\text { análisis } 1\end{array}$} & Correlación de Pearson & 1 &, 000 &, 000 \\
\hline & Sig. (bilateral) & & 1,000 & 1,000 \\
\hline & $\mathrm{N}$ & 22 & 22 & 22 \\
\hline \multirow{3}{*}{$\begin{array}{l}\text { Puntuación de factor } 2 \text { para } \\
\text { análisis } 1\end{array}$} & Correlación de Pearson &, 000 & 1 &, 000 \\
\hline & Sig. (bilateral) & 1,000 & & 1,000 \\
\hline & $\mathrm{N}$ & 22 & 22 & 22 \\
\hline \multirow{3}{*}{$\begin{array}{l}\text { Puntuación de factor } 3 \text { para } \\
\text { análisis } 1\end{array}$} & Correlación de Pearson &, 000 &, 000 & 1 \\
\hline & Sig. (bilateral) & 1,000 & 1,000 & \\
\hline & $N$ & 22 & 22 & 22 \\
\hline
\end{tabular}

Lo que muestra un nivel alto de significancia entre los nuevos 3 factores y ratifica que la gestión del conocimiento en empresas y organizaciones del clúster de TI del eje cafetero, se está desarrollando desde la generación, la identificación y la aplicación de conocimiento.

\section{CONCLUSIONES}

De los resultados mostrados, de su análisis y de su discusión, se pueden obtener las siguientes conclusiones, sobre la gestión del conocimiento en el clúster de TI del Eje Cafetero de Colombia:

1. Aunque se está apoyando el desarrollo de competencias para la gestión del conocimiento, esto no se ve reflejado en las demás categorías de análisis, lo que muestra que aún no se ha llevado a términos prácticos y reales, dados los resultados de la innovación y el aprendizaje.

2.- En lo referente a prácticas para la gestión del conocimiento, es necesario avanzar en su consolidación, toda vez que los resultados fueron bajos en su compartición y aplicación, dado que se usan diversas técnicas, pero no se comparten en la comunidad empresarial.

3.- Se propone a las empresas incorporar la gestión del conocimiento para innovar mediante el manejo direccionado de procesos de conocimiento (identificar, compartir, generar, retener y aplicar conocimiento), formando al capital humano en gestión de la información y la documentación, en gestión de la innovación y el cambio, en gestión del aprendizaje organizacional, en diseño de herramientas digitales y en gestión de la comunicación.

\section{REFERENCIAS}

Aguilera, L., González, M., y Maldonado, G. Capital intelectual: la innovación, indicador del capital estructural de empresas de sectores estratégicos en aguas calientes. Memorias XXI Congreso Latinoamericano sobre Espíritu Empresarial, 249-270, Cali-Colombia, 6 al 8 de abril (2011).

Alonso, L. F. El imaginario Managerial. Política y sociedad: 43, 127-151, (2006).

Álvarez, F. C. Riqueza Natural de la Nación, 1ª edición, 180-195. Torre Azul, Lima, Perú, (2004).

Andrade, J., Ares, R., García, J., Pazos, S., Rodriguez, y A. Silva. Formal conceptualisation as a basis for a more procedural knowledge management. Decision Support Systems, 45, 164-179, (2008).

Barnes, J., y Liao, Y. The effect individual network, and collaborative competencies on the supply chain management system. Production Economics, 888-899, (2012).

Bernal, C., Turriago, A., y Sierra, H. Aproximación a la medición de la gestión del conocimiento de la gestión del conocimiento. AD-MINISTER, 16, 30-49, (2010).

Briceño, M., y Bernal, C. Estudios de caso sobre la gestión del conocimiento en cuatro organizaciones colombianas líderes en penetración de mercado. Estudios gerenciales, 173-193, (2010). 
Bueno, E., Enfoques principales y tendencias en dirección del conocimiento (Knowledge management). Dirección de Conocimiento: Desarrollo Teórico y aplicaciones, 21-54, (2003)

Bueno, E., Gestión del Conocimiento: desarrollos teóricos y aplicaciones. 2a edición, 25-38. Ediciones La Coria, Cáceres, (2003).

Bingi, R.,, Khazanchi D., y S. Yadav, A framework for the comparative analysis and evaluation of knowledge representation schemes. Information Processing y Management, 20, 3, 233-247, (1995).

Carrillo., A Global Knowledge Agenda based on Capital Systems (2005), http://www.sistemasdeconocimiento.org. Acceso: 3 de Mayo (2011).

Capó-Vicedo, J., Tomás-Miquel J., y Expósito-Langa, M., La Gestión del Conocimiento en la Cadena de Suministro. Análisis de la Influencia del Contexto Organizativo. Revista Información Tecnológica, 18 (1), 127-136, (2007).

CEN., European Commitee for standardization (2004), http://cenftp1.cenorm.be/public/cwas/eeurope/KM/CWA14924-01-2004-Mar.pdf. Acceso: 4 de Julio (2012).

Chen, T. A., multiple-layer knowledge management system framework considering user knowledge privileges. International Journal of software engineering and knowledge engineering, 361-387, (2008).

Chiva-G, R., Repercussions of complex adaptive systems on product design management. Technovation, 707-711, (2004).

Corbin, R.,, C., Dumbar, y Q. Zhu. A three-tier knowledge management scheme for software engineering support and innovation. The Journal of Systems and Software, 80, 1494-1505, (2007).

Daedalus., Data, Decisions And Language (2009), http://www.daedalus.es/. Acceso: 1 de Junio (2011)

DelMoral, A., Pazos, J., Rodríguez, E., Rodríguez, P., y Suarez, S. Gestión del Conocimiento. 1ª edición, 4860. Thomson, Madrid, (2007).

DNP., Dirección Nacional de Planeación (2005), http://www.dnp.gov.co/. Acceso: 22 de noviembre (2011).

Escorsa., De la vigilancia tecnológica a la inteligencia competitiva. 1ª edición, 9-19. Pearson, Madrid, (2001).

EUROSTAT, O., Manual de Oslo: Guía para la recogida e interpretación de datos sobre innovación. $3^{a}$ edición, 101-142. Grupo Tragsa, París, (2006).

Garzón C., M. A., Aproximaciones a la gestión del conocimiento en empresas Colombianas. Universidad Empresa, 232-256, (2006).

Hautala, J., International academic knowledge creation and ba. Knowledge Management Research y Practice, 4-12, (2011).

Johannessena, J.-A., Olsena, B., y Olaisen, J., Intellectual capital as a holistic management philosophy: a theoretical perspective. International Journal of Information Management, 25, 151-171, (2005).

Joyanes, L., Cuestas, a., y López, M., Caracterización de la industria del software en el triángulo del café, Entre ciencia e ingeniería, 76-87, (2010).

Klavans, R., Technology Strategy and Competitive Intelligence. Global perspectives on competitive intelligence, 129-130. (1993).

Knudsen, C., Theories of the firm, strategic management, and leadership. $1^{\mathrm{a}}$ edición, 179-203. Kluwer Academic Publishers, London, (1995).

Kok, A., Intellectual Capital Management as Part of Knowledge Management Initiatives at Institutions of Higher Learning, The Journal of Knowledge Management, 5 (2),181-192, (2007). 
Lobato, V., Caracterización del conocimiento en PYMES que realizan proyectos TIC: un modelo de análisis y valoración, Tesis de doctorado, Depto. de explotación y prospección de minas, Universidad de Oviedo, Oviedo, España, (2008).

López, L., y Calderón, G., Análisis de las dinámicas culturales al interior de un clúster empresarial, Estudios gerenciales, 13-37, (2006).

López, M., Ciudadanía Digital, un modelo de implantación en la región de Manizales y Caldas, Tesis de doctorado, Depto. de Ingeniería, Universidad pontificia de Salamanca, Madrid, (2010).

McBriara, I.,, C., Smith, G., Bainc, P., Unsworthd, S.. Magrawe, y F. Gordon. Risk, gap and strength: key concepts in knowledge management. Knowledge-Based Systems, 16, 29-36, (2003).

Marr, B., Measuring and benchmarking intellectual capital. International Journal, 11(6), 559-570, (2004).

Martínez, M., Desarrollo de un modelo de gestión del conocimiento en la cadena de suministro de la industria agroalimentaria, Tesis de doctorado, Depto de Ingeniería de Organización, Universidad Politécnica de Madrid, Madrid, España, (2011).

Marulanda, C., y López, M., Cultura organizacional y gestión del cambio y de conocimiento en las organizaciones de Caldas, Revista virtual de la Universidad Católica del Norte, 117-139, (2011).

Matilla, M., y Chalmeta, R., Metodología para la Implantación de un Sistema de Medición, Información Tecnológica, 119-126, (2007).

Matos, G., Chalmeta, R., y Coltell, O., Metodología para la Extracción del Conocimiento Empresarial a partir de los Datos. Información Tecnológica, 17, 2, 81-88, (2006).

Meisel, S., Bermeo, H., y Oviedo, L., Generación de valor a través de la gestión estratégica del conocimiento, innovación y la mejor continua, Scientia et Technica, 165-170, (2006).

Morales, M., Acumulación de conocimiento, innovación y competitividad en aglomeraciones industriales, Revista de la Facultad de Ciencias Económicas, 19-53, (2010).

Nahapiet, J., y Ghoshal, S., Social capital, intellectual capital, and the organizational advantage. The Academy of Management Review, 242-266, (1998).

OCDE, Industrial Competitiveness. 1a edición, 75-89. OCDE, París, (1997).

Palop, F. V., Vigilancia Tecnológica e Inteligencia Competitiva. 1ª edición, 85-94. COTEC, Madrid, (1999).

Paniagua, E., La Gestión Tecnológica del Conocimiento. 1ª edición, 39-48. U. de Murcia, Murcia, (2007).

Patria, L., El clúster de TI del eje cafetero (2011), http://www.lapatria.com. Acceso: 9 de enero (2011)

Peña, T. J., Modelo integral de gestión del conocimiento, $1^{a}$ edición, 105-116. Universidad de Burgos, Burgos, España, (2006).

Pérez, D., Tecnologías de información para la gestión del conocimiento, Intangible Capital, 31-59, (2007).

Porter, M., La ventaja competitiva de las naciones, $1^{a}$ edición, 48-57. Vergara, Buenos Aires, (1991).

Prassl, W.,, J., Peden, y K. Wong. A process-knowledge management approach for assessment and mitigation of drilling risks. Journal of Petroleum Science and Engineering 49, 142-161, (2005).

Proexport., Software y Servicios de TI, documento, Ministerio de Comercio de Colombia, Bogotá, (2011).

Riesco, M., El negocio es el conocimiento, 1ª edición, 150-169. Díaz de Santos, Madrid, (2006).

Romero, D., Mathison, L., y Rojas, D., Una propuesta de gestión de conocimiento para la pequeña y mediana empresas, Revista da Micro y Pequeña Empresa, 120-143, (2009). 
Sanabria, S., Morales, M., y Arias, M. Conocimiento, innovación y competitividad en aglomeraciones empresariales, Facultad de Ciencias económicas, 19-53. (2010).

Sánchez, A., Modelo para la medición del capital intelectual de territorios insulares, Tesis de doctorado, Depto. De ingeniería, Universidad de las Palmas de Gran Canaria, Gran canaria, España, 75-85. (2003).

Shin, M., Holden, T., y Schmidt, R., From knowledge theory to management practice: towards an integrated approach, Information Processing and Management, 37, 335-355, (2001).

Tena, J. C., Inteligencia Competitiva y Vigilancia Tecnológica, $1^{\mathrm{a}}$ edición, 64-75. Emecom ediciones, Barcelona, (2006).

Toffler, A., El cambio del poder, 1ª edición, 91-97. Editorial Plaza y Janés, Barcelona, (1990).

Torres, M., A procedure to design a structural and measurement model of Intellectual Capital: An exploratory study, Information and management, 617-626, (2006).

Valencia., D., El sector de software en Colombia, documento de la Cámara de Comercio, Bogotá, (2010).

Zaintek, Guía de Vigilancia Tecnológica: Sistema de información estratégica en las pymes, $1^{\mathrm{a}}$ edición, 2641. Zaintek, Bilbao, España, (2003). 